\title{
Rapid Identification of $M$. tuberculosis Complex and Non Tuberculous Mycobacterium in Extra Pulmonary Tuberculosis using MGIT 320 Liquid Culture System and MPT64 Antigen Test
}

\author{
Mahwish Jawaid, Qursheed Sultana*, Ajaz Hussain, Md Khaleel and \\ Maimoona Mustafa
}

Department of Microbiology, Deccan College of Medical Sciences, Hyderabad, India

*Corresponding author

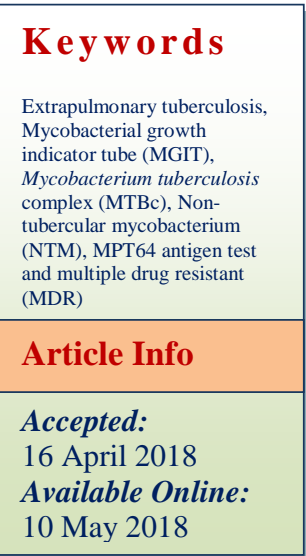

Tuberculosis is a major cause of morbidity and mortality worldwide. Mycobacterium tuberculosis has infected one third of world's population and causes 8.8 million new cases

\section{Introduction}

population a
Tuberculosis is a major cause of morbidity and mortality worldwide. Mycobacterium tuberculosis has infected one third of world's population and causes 8.8 million new cases with approximately 1.1 million deaths each year. A definitive diagnosis of TB can only be made by culturing Mycobacterium tuberculosis organisms from a specimen obtained from the patient. However diagnosing EPTB remains challenging because clinical samples obtained from relatively inaccessible sites may be paucibacillary, decreasing the sensitivity of diagnostic tests. The present study was initiated to determine the prevalence of Mycobacterium tuberculosis, Non Tuberculous Mycobacterium and its resistance to first line Anti-Tubercular drug from extra pulmonary samples. A total of 265 extra pulmonary samples were included in this study. All the samples screened by Zeihl-Neelsen AFB microscopy, were subjected to liquid culture using Mycobacterium Growth Indicator Tube (MGIT-320). Positive cultures were differentiated into Mycobacterium tuberculosis complex (MTBc) or Non-tubercular mycobacterium (NTM) by immunochromatography assay using MPT-64 antigen. Drug susceptibility testing of MTBc isolates done using MGIT-320. $32 \mathrm{MTBc}$ and 7(NTM) grown in MGIT320 system were examined by the rapid MPT64 antigen detection. Among 32MTBc isolates 26 were sensitive to all the drugs and 6 were resistant to one or more drugs. Multiple drug resistant (MDR) isolates were 2. The susceptibility results were available within 11-13 days, thus within an average of 3 weeks of time complete results could be produced. We reported $6.6 \%$ prevalence of MDRTB in EPTB at our tertiary care hospitals in southern India which is little lesser compared to other studies, reflecting better TB control programme. The prevalence of NTMs is also increasing, rising the importance of their recognition. with approximately 1.1 million deaths each year. The two types of clinical manifestation of tuberculosis (TB) are pulmonary TB (PTB) and extrapulmonary TB (EPTB). The former is most common. EPTB refers to TB involving organs other than the lungs (e.g., pleura, 
lymph nodes, abdomen, genitourinary tract, skin, joints and bones, or meninges). A patient with both pulmonary and EPTB is classified as a case of PTB. For example, miliary TB is classified as PTB because there are lesions in the lungs. On the other hand, tuberculous intrathoracic lymphadenitis (mediastinal and/or hilar) or tuberculous pleural effusion, without radiographic abnormalities in the lungs, constitutes a case of EPTB (World Health Organization, 2007). A definitive diagnosis of TB can only be made by culturing Mycobacterium tuberculosis organisms from a specimen obtained from the patient. However diagnosing EPTB remains challenging because clinical samples obtained from relatively inaccessible sites may be paucibacillary, decreasing the sensitivity of diagnostic tests. Since the conventional smear microscopy has a low sensitivity with a range of $0 \%-40 \%$, negative results cannot exclude the presence of TB. The reported yields of mycobacterial culture vary from $30 \%$ up to $80 \%$, but it usually takes $2-8$ weeks to receive the results, which is too slow to help treatment decisions (Tortoli et al., 1999). The importance of early diagnosis and correct etiological identification of extra pulmonary tuberculosis (EPTB) need not be overemphasised, since treatment is different for Mycobacterium tuberculosis and atypical Mycobacteria (non-tuberculous Mycobacteria, NTM). World Health Organization has given guidelines for low and medium income countries for use of liquid culture systems and drug sensitivity testing for tuberculosis work (World Health Organization, 2007). Automated non-radiometric systems for accelerated isolation of Mycobacterium tuberculosis complex (MTBC), being expensive, are available only in select centre's in India and third-world countries. However, most laboratories still depend upon conventional techniques, thus resulting in an extended reporting time of 4-5 weeks. The MGIT is a liquid broth medium that is known to yield better recovery and faster growth of mycobacteria. In addition to Middlebrook 7H9 liquid media, the MGIT tube contains an oxygen-quenched fluorochrome. It detects oxygen consumption induced by growing micro-organisms (Abe et al., 1999). There are a few published reports on the evaluation of BACTEC MGIT 320 on extrapulmonary samples. An innovative rapid kit, MPT64-ICT, to detect an established marker of MTBC, the MPT64 antigen, by immunochromatography test (ICT) developed by Japanese scientists (Maurya et al., 2012) found universal acceptance due to its simplicity, accuracy and rapidity. Indian reports on EPTB in general (Vadwai et al., 2012; Fras Er. Wares et al., 2005; Becton, 2004) and the use of rapid kits for confirmation of MTBC in particular are few.

The present study was initiated to determine the prevalence of Mycobacterium tuberculosis, Non Tuberculous Mycobacterium and its resistance to first line Anti-Tubercular drug from extra pulmonary samples among patients attending a tertiary care hospital in Hyderabad.

\section{Materials and Methods}

\section{Study design and period}

The study was carried out in the clinical Microbiology laboratory of a tertiary care hospital in Hyderabad during the period January 2016 to December 2017. Our Institutional Human Ethics Committee scrutinized and approved this research. Patients' informed consent was obtained before collection of specimens.

\section{Study population}

A total of 265 single clinical specimens of patients suspected for EPTB were included in the study. The clinical samples included 
Lymph node aspirate (15), pleural fluid (159), CSF (11), pus (16), ascitic fluid (39), E.T secretions (8), urine (6) pericardial fluid (7), Semen (1), and peritoneal fluid (3).

\section{Acid fast bacilli smears}

Smears were prepared from each sample, stained by Ziehl Neelsen method and examined for presence of AFB with a light microscope.

Decontamination and processing of the samples

All specimens were liquefied and decontaminated by the standard N-acetyl-Lcysteine, sodium hydroxide method $(\mathrm{NaOH}-$ NALC). After $15 \mathrm{~min}$ holding at room temperature, specimens were neutralized with phosphate buffer saline (PBS, pH 6.8) and centrifuged in cold centrifuge at $4500 \mathrm{rpm}$ for $20 \mathrm{~min}$ at $10^{\circ} \mathrm{C}$. The pellets were resuspended in $1.5 \mathrm{ml}$ of sterile phosphate buffer and collected for further analysis.

\section{BACTEC MGIT 320 liquid media}

The BBL MGIT tube was inoculated by 0.5 $\mathrm{ml}$ of the decontaminated and concentrated specimen suspension. It contained $7 \mathrm{~mL}$ of modified middlebrook $7 \mathrm{H} 9$ broth enrichment with albumin, dextrose and catalase (BBL MGIT OADC) and an antibiotic mixture consisting of polymyxin $\mathrm{B}$, amphotericin $\mathrm{B}$, nalidixic acid, trimethoprim, and azlocillin (BBL MGIT PANTA).

After inoculation, the tubes were loaded in the BACTEC MGIT 320 instrument and incubated up to 42 days at $37^{\circ} \mathrm{C}$. Culture vials are monitored hourly by the instrument. All the positive tube was further confirmed by $\mathrm{ZN}$ staining, subculturing on blood agar plate. The TTD of mycobacteria was based on the date of the earliest instrumental indication of positivity.

(MGIT, 2007 http://www.standardia.com/en/home/product/r apid/infectious-disease/TB_Ag_MPT64.html)

\section{Identification morphological and biochemical identification}

For differentiation of M. tuberculosis complex and NTM, a commercially available kit was used, the BD MGIT MTBc identification test (TBc ID). It is a rapid chromatographic immunoassay for the qualitative detection of $M$. tuberculosis complex antigen MPT64 from AFB smearpositive BD MGIT tubes. The assay is performed according to the manufacturer's instructions (Kanade et al., 2012; Kumar et al., 2011; Kandhakumari et al., 2015).

Briefly, $100 \mu 1$ of mixed and vortexed culture fluid from AFB positive MGIT tubes were transferred to sample window of the cassette. The results of ICT were read within 15 minutes. Positive test had two red to purple bands, one for internal control and the second line for the test. Negative had only one band in internal control slot. Strong or light bands with any intensity were considered to be positive.

MGIT tubes showing non-acid fast bacilli and/or fungi were excluded from MPT64 Ag test (http://www.standardia.com/en/home/ product/rapid/infectious-disease/TB_Ag_ MPT64.html).

\section{BACTEC MGIT 960 liquid media DST}

MTBc isolates was further tested for the first line drugs in BACTEC MGIT 320. Conc. of various drugs used was - streptomycin (STR) - $1 \mu \mathrm{g} / \mathrm{ml}$, isoniazid (INH) - $0.1 \mu \mathrm{g} / \mathrm{ml}$, rifampicin (RIF) - $1 \mu \mathrm{g} / \mathrm{ml}$, ethambutol (ETB)$5 \mu \mathrm{g} / \mathrm{ml}$. Drug susceptibility was reported when the growth control units reached 400 as indicated by the instrument. (MGIT, 2007) 


\section{Control strains}

Reference strains of H37Rv and Mycobacterium fortuitum were included as positive and negative controls, respectively.

\section{Results and Discussion}

Total two hundred and sixty five (265) samples from patients with clinical/radiological suspicion of Extrapulmonary Tubercular infection were included in this study. Type of samples and their isolation rate by various methods is shown in Table 1.

Out of the total 265 samples, 39 (14.72\%) samples were positive out of which 32 were MTBc (Mycobacterium tuberculosis complex) and 7 were NTM (Non-Tubercular Mycobacterium sp) (Table 2, Figure 1). Out of the total 265 samples, 145 were male and 120 were female patients (Table 3). Majority of the positive samples, both in males and females, belong to the age group of 20-40 years followed by 40-60 years (Figure 2). Among positives isolates $6(15.4 \%)$ were positive for AFB on Direct smear examination. The average TTD was 16 days for MGIT 320 with the extremity from 8 to 38 days. For smearpositive specimens, the mean turnaround time was 14 days and for smear-negative specimens, the same was 19 days. The rate of contamination in liquid medium using BACTEC MGIT-320 was $6.2 \%$.

Antibiotic susceptibility testing for all the first-line drugs was performed on 32 strains of MTBc using BACTEC MGIT320 system. 26 strains were sensitive to all drugs and 6 were resistant to one or more drugs. Isolates resistant to both INH and RIF i.e. MDR-EPTB was 2 (Figure 3).

The reported prevalence of Extrapulmonary Tuberculosis (EPTB) and drug-resistant EPTB was found variable in different studies from around the world and in our country. In our study, out of the 265 extra pulmonary samples, $39(14.7 \%)$ were culture positive out of which 32 (12.1\%) were MTBc (Mycobacterium tuberculosis complex) and 7 (2.6\%) were NTM (Non-Tubercular Mycobacterium sp). similar study done by Kandhakumari et al., (2015) gave the isolation rate of $10.2 \%$, out of which MTBc strains were $9.1 \%$ and NTM strains were $1.1 \%$. (Jain et al., 2002) Number of AFB present in clinical specimens from EPTB cases is fewer than in sputum. Since decades direct smear examination of AFB by Z-N method was the only method to relay on for initiating early treatment. But as the literature (Chakravorty et al., 2005; Makeshkumar et al., 2014; Sharma and Mohan, 2004; Chihota et al., 2010) reflect it has very less sensitivity and possibility of false positives is also increased as the attenuated or dead bacilli can't be differentiated. In our study, among 39 positives isolates $6(15.4 \%)$ were positive for AFB on Direct smear examination. Smear microscopy for AFB is rapid but less sensitivity and cannot discriminate between MTBC and NTM (World Health Organization, 2007). The automated liquid systems have improved the recovery of Mycobacterium from smear negative samples (World Health Organization, 2015), but there is still a need for rapid identification of MTB isolates. For treatment of Tuberculosis it is necessary to differentiate $M$. tuberculosis from NTM (NonTubercular Mycobacterium sp). An innovative rapid kit, MPB64-ICA, to detect an established marker of MTBC, the MPT64 antigen, by immunochromatography test (ICT) developed by Japanese scientists (Maurya et al., 2012) found universal acceptance due to its simplicity, accuracy and rapidity. Kannade et al., (2012) from Bombay (Mumbai) who examined 165 isolates (125 MTB; 30 NTM; 10 Non-Mycobacterial species) and observed sensitivity of $99.19 \%$ and $100 \%$ values for 
specificity, positive predictive value (PPV) and negative predictive value (NPV) for the rapid MPT64 antigen detection kits in comparison to conventional methods. Vadwai et al., (2012) from Bombay analysed 394 strains from 280 pulmonary and 114 EPTB samples (388 MTB; 6 NTM) with similar result, i.e. $99.4 \%$ sensitivity and $100 \%$ specifi city. Kumar et al., (2011) from Mysore, Karnataka, analysed 77 isolates (55 MTB; 10 NTM; 12 Non-Mycobacterial species) recorded $100 \%$ results for all four parameters.

Table.1 Distribution of various extrapulmonary sample in patients

\begin{tabular}{|l|c|c|}
\hline Type of Sample & No of cases & Positive \\
\hline Pleural fluid & 159 & 17 \\
\hline Pus & 16 & 4 \\
\hline Ascitic fluid & 39 & 8 \\
\hline ET Secretion & 8 & 1 \\
\hline CSF & 11 & 1 \\
\hline Urine & 6 & 1 \\
\hline Pericardial fluid & 7 & 0 \\
\hline Lymph Node & 15 & 7 \\
\hline Peritoneal fluid & 3 & 0 \\
\hline Semen & 1 & 0 \\
\hline Total & 265 & 39 \\
\hline
\end{tabular}

Table.2 Distribution of culture positive cases

\begin{tabular}{|c|c|c|}
\hline No. of cases studied & No. of positive cases & No. of negative cases \\
\hline 265 & $39(14.72 \%)$ & $226(85.28 \%)$ \\
\hline
\end{tabular}

Table.3 Gender distribution of patients and percentage of positive sample

\begin{tabular}{|c|c|c|}
\hline Gender & No. Of collected samples (\%) & Organisms isolates (\%) \\
\hline Male & $145(54.72)$ & $21(7.93)$ \\
\hline Female & $120(45.28)$ & $18(6.79)$ \\
\hline Total & 265 & $39(14.72)$ \\
\hline
\end{tabular}


Fig.1 MTBC and NTM positive samples

\section{Distribution of Positive Samples $(n=39)$}

MTBC $32 \quad$ NTM 9

$22 \%$

Fig.2 Age and sex distribution of positive cases

\section{Age and sex wise distribution of positive cases in various age groups}

nale $\quad$ Female

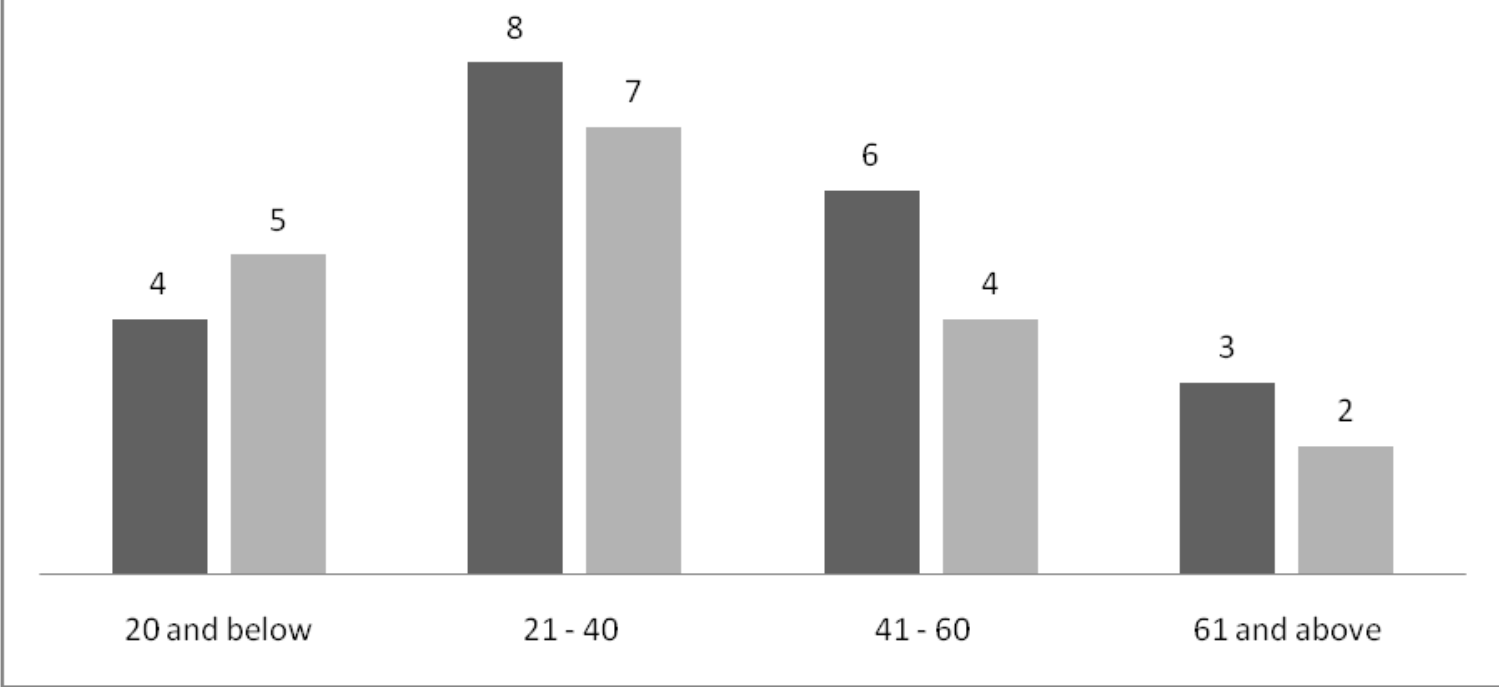


Fig.3 Distribution of sensitive and resistant cases

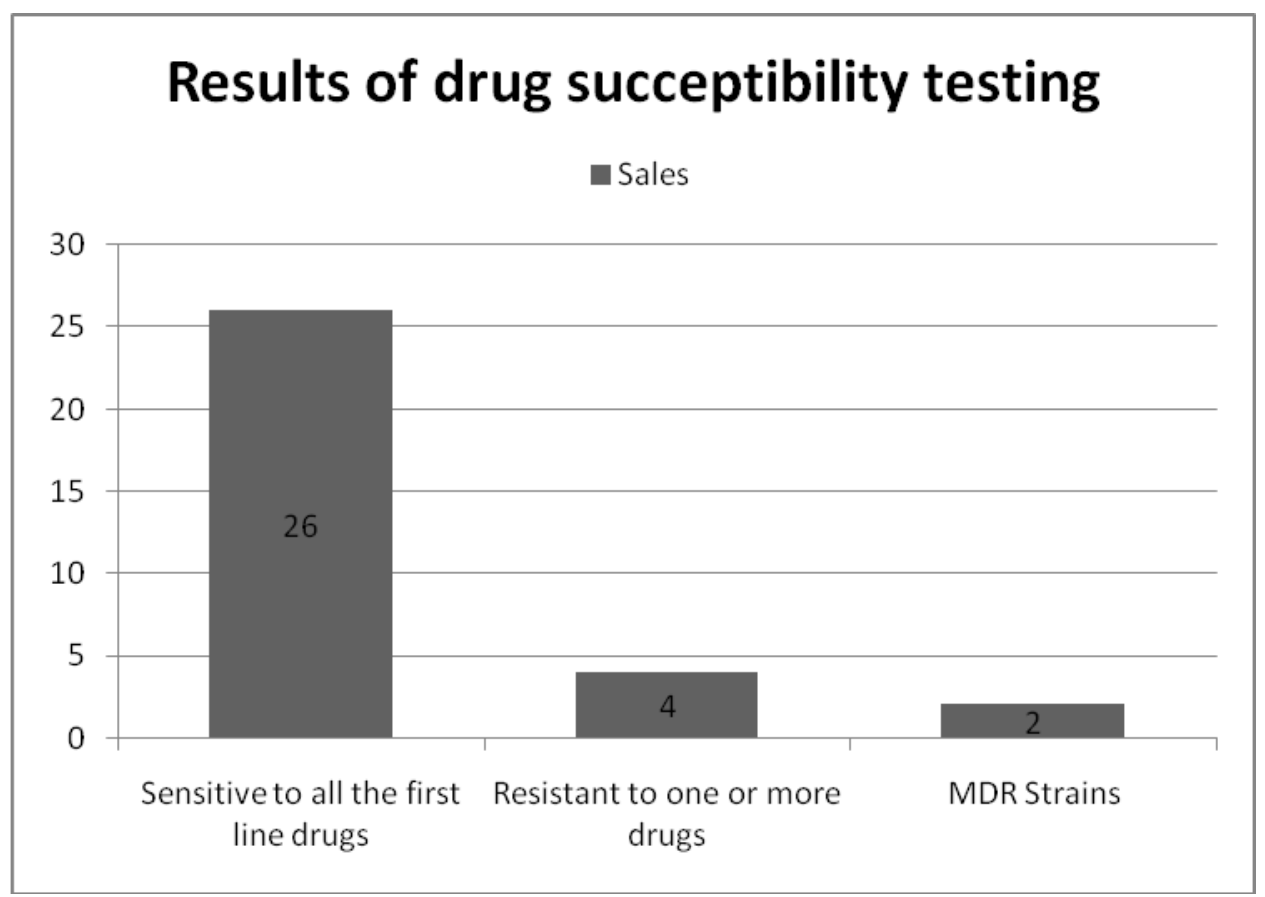

India is among the four countries that have the largest number of estimated cases of MDR-TB (China, India, the Russian Federation and South Africa) (Gurung et al., 2010). Detection of resistance at the genetic level by molecular methods, identifying mutations at certain loci, although promising, is still far from finding its place among the phylogenetic techniques. The major limitation of detecting resistance by molecular methods is the presence of multiple resistance mechanisms in majority of anti-mycobacterial drugs. (Abe et al., 1999) Therefore phenotypic susceptibility testing remains the trusted method. Among the phenotypic methods, the use of liquid media is the best available possibility of speeding up the testing. Therefore the sensitivity of first line ATTs was done using BACTEC MGIT-320. In our study, out of 32 positive isolates, 26 $(81.2 \%)$ were sensitive to all drugs and 6 $(18.7 \%)$ were resistant to one or more drugs. Isolates resistant to both $\mathrm{INH}$ and RIF i.e. MDR-EPTB was 2 (6.6\%). Previous studies reported a high rate of MDR- EPTB cases
(12.5\%) from Nepal, (Sachdeva et al., 2002) (10\%) from Delhi- India, and $13.5 \%$ from Lucknow (Vadwai et al., 2012). We reported $6.6 \%$ prevalence of MDR-TB in EPTB at our tertiary care hospitals in southern India which is little lesser compared to other studies, reflecting better TB control programme.

The susceptibility results were available within 11-13 days, thus within an average of 3 weeks of time complete results including isolation, identification and drug susceptibility testing of isolate could be produced.

We conclude that the MGIT 320 system provides higher isolation rate with rapidity and better sensitivity and specificity of both MTBc and NTMs from a variety of extrapulmonary samples. Recovery of the MTBc and NTM isolates was more accurate and rapid when MGIT960 system combined with rapid ICT kit which detects MPT64 Ag in 15 minutes thus we advise combined use. The prevalence of NTMs is also increasing, rising 
the importance of their recognition. In addition to pulmonary TB, EPTB also need surveillance programs and rapid drug susceptibility testing technique to recognize early and exact burden of MDR- EPTB, which is necessary for the successful management and control of MDR-TB.

\section{References}

Abe C, Hirano K, Tomiyama T. Simple and rapid identification of the Mycobacterium tuberculosis complex by immunochromatographic assay using anti-MPB64 monoclonal antibodies. J Clin Microbiol 1999; 37: 3693-7.

Becton, Dickinson and Company (2004) BACTEC MGIT 960 System User's Manual. Sparks, MA.

Chakravorty S, Sen MK, Tyagi JS. Diagnosis of Extrapulmonary Tuberculosis by Smear, Culture and PCR Using Universal Sample Processing Technology. J Clin Microbiol. 2005; 43(9): 4357-4362.

Chihota, V.N., Grant, A.D., Fielding, K., Ndibongo, B., van Zyl, A., Muirhead, D., et al., (2010) Liquid vs. Solid Culture for Tuberculosis: Performance and Cost in a Resource-Constrained Setting. International Journal of Tuberculosis and Lung Disease, 14, 1024-1031.

Fras Er. Wares, Balasubramanian R, Mohan A Sharma SK. Extra pulmonary tuberculosis, management and control. In: Agarwal SP, Chauhan LS, editors. Tuberculosis Control in India. New Delhi: Elsevier; 2005: 95-114.

Gurung R, Bhattacharya SK, Pradhan B, Gurung S, Singh Y. Phenotypic characterisation and drug sensitivity testing of mycobacteria isolated from extra-pulmonary tuberculosis. Kathmandu Univ Med J. 2010; 2010: 57-61
Jain A, Bhargava A, Aggarwal SK. A comparative study of two commonly used staining techniques for acid fast bacilli in clinical specimens. Ind $\mathrm{J}$ Tub. 2002; 49: 161.

Kanade, S., Nataraj, G., Suryawanshi, R. and Mehta, P. (2012) Utility of MPT64 Antigen Detection Assay for Rapid Characterization of Mycobacteria in a Resource Constrained Setting. Indian Journal of Tuberculosis, 59, 92-96.

Kandhakumari, G., S Stephen et al., Extra pulmonary tuberculosis: Rapid identifi cation of Mycobacterium tuberculosis grown in Mycobacterium growth indicator tube 960 and LowensteinJensen media, employing Standard diagnostics Bioline Mycobacterium tuberculosis protein 64 antigen detection kit. Indian Journal of Medical Microbiology, (2015) 33(Supplement 1): S122-25.

Kumar, V.G., Urs, T.A. and Ranganath, R.R. (2011) MPT64 Antigen Detection for Rapid Confirmation of $M$. tuberculosis Isolates. BMC Research Notes, 4, 79. http://dx.doi.org/10.1186/1756-0500-479

Makeshkumar V, Madhavan R, Narayanan S. Polymerase chain reaction targeting insertion sequence for the diagnosis of extrapulmonary tuberculosis. Indian $\mathbf{J}$ Med Res., January 2014; 139: 161-166.

Maurya AK, Nag VL, Kant S, Kushwaha RA, Kumar M, Mishra V, et al., Evaluation of an immunochromatographic test for discrimination between Mycobacterium tuberculosis complex and non tuberculous mycobacteria in clinical isolates from extra-pulmonary tuberculosis. Indian J Med Res 2012; 135: 901-6.

MGIT for BACTEC ${ }^{\mathrm{TM}}$ (2007) MGIT 960'TM TB System Manual.

Sachdeva R, Gardre DV, Talwar V. Characterization and drug susceptibility 
patterns of extra-pulmonary mycobacterial isolates. Indian $\mathbf{J}$ Med Res., 2002; 115: 102-5.

Sharma SK, and Mohan A. Extrapulmonary tuberculosis. Indian J Med Res., 2004; 120: 316-353.

TB Antigen MPT64. http://www.standardia. com/en/home/product/rapid/infectiousdisease/TB_Ag_MPT64.html

Tortoli E, Cichero P, Piersimoni C, Simonetti MT, Gesu G, et al., (1999) Use of BACTEC MGIT 960 for recovery of mycobacteria from clinical specimens: multicenter study. J Clin Microbiol 37: 3578-3582.

Vadwai V, Sadani M, Sable R, Chavan A, Balan K, Naik A, et al., Immunochromatographic assays for detection of Mycobacterium tuberculosis: What is the perfect time to test? Diagn Microbiol Infect Dis 2012; 74: 282-7.

World Health Organization. Global tuberculosis report 2015. Geneva: TB publications. Available from www.who.int/tb/publications/global_rep ort $/ 2015 / 22$.

World Health Organization. Use of liquid TB culture and drug susceptibility testing (DST) in low and medium income countries: Summary report of the expert group meeting on the use of liquid culture media. Geneva, Switzerland: WHO 2007. Available from: http://www.who.int/tb/laboratory/use_of _liquid_tb_culture_summary_report.pdf Canadian Thoracic Society and The Public Health Agency of Canada and Licensors. Canadian tuberculosis standards. 7th ed. Ottawa: Public Health Agency of Canada; 2013.

\section{How to cite this article:}

Mahwish Jawaid, Qursheed Sultana, Ajaz Hussain, Md Khaleel and Maimoona Mustafa. 2018. Rapid Identification of $M$. tuberculosis Complex and Non Tuberculous Mycobacterium in Extra Pulmonary Tuberculosis using MGIT 320 Liquid Culture System and MPT64 Antigen Test. Int.J.Curr.Microbiol.App.Sci. 7(05): 2036-2044. doi: https://doi.org/10.20546/ijcmas.2018.705.239 